\title{
A Real time 3D video CMOS sensor with time gated photon counting
}

\author{
Alexis Matwyschuk, Emmanuel Bacher, Nicolas \\ Metzger \\ Institut franco-allemand de recherches de Saint-Louis \\ Saint-Louis, France \\ Alexis.Matwyschuk@isl.eu
}

\author{
Wilfried Uhring, Jean-Pierre Le Normand, Octavian \\ Maciu, Imane Malass, Norbert Dumas \\ ICube Laboratory \\ University of Strasbourg and CNRS \\ Strasbourg, France \\ Wilfried.uhring@unistra.fr
}

\begin{abstract}
The real time acquisition of a 3D scene has been recently democratized by video game consoles. The most popular approaches such as the structure-light and the stereo vision are appropriate for short distances but they are not well suited for long time measurements in the range of a hundred meters up to a few kilometers or a scattering environment. In this case, the time of flight method is more convenient but is limited by the heavy and expensive hardware requirement for military applications. Indeed, a strong laser illumination and a time gated intensified camera is generally used to carry out this kind of measurement. The signal reflected at a long distance is so weak that very few photons reach the camera, thus using an image intensifier is mandatory. Moreover, the acquisition time and processing is quite long as several laser pulse are required for distance measurement and several frame of distance measurement are required to build a $3 D$ scene. As a consequence, real time measurement cannot be achieved at this time. The paper describes a novel CMOS imager based on time gated single photon avalanche diode in order to detect the reflected light and accomplish distance memorization. The processing is fully parallelized and embedded within the pixel. The pixel includes 46 transistors, operates independently and has a size of $36 \times 43 \mu \mathrm{m}^{2}$. As a result, the readout of the sensor generates the 3D images immediately in real time.
\end{abstract}

Keywords-Real time $3 D$ video, SPAD, Photon couting, Time gating

\section{INTRODUCTION}

Over the past few years, more and more active imaging systems have been used and tested [1][2]. The potential of such systems in the fields of long-range observation [3] and in the vision through scattering environments [4] has shown clear advantages. Following a patent filed concerning a process of 3D imaging [5], this paper described a CMOS sensor based on this new process. This matrix active imaging system named MAITODIC (Matrix Active Imaging system of direct conversion TOmographic Data Into a Color image 3D) directly converts the tomographic data of a scene into a 3D image where every depth slice of the scene is represented by a color (according to a predefined color or depth code). Contrary to systems measuring the time of flight, this system assumes that the time of flight is known and only the information arriving at this precise moment is taken into account. This eliminates the backscattering effects during the propagation of the light through scattering environments, like rain, snow, fog, mist or smoke, which distort the distance information.

\section{THE MAITODIC SENSOR}

\section{A. Concept of the $3 D$ imaging}

Active imaging is a visualization technique using an image sensor array and its own illumination source to display directly a two-dimensional image of the scene. According to the synchronization between the illumination and the recording, it is possible to improve the visibility through the scattering environments. The use of light pulse sources like lasers and triggered intensified cameras with low exposure times enables range gating. More exactly, it allows us to visualize a space slice of the scene at a certain distance according to the laser pulse and to the acquisition time. In full field active imaging, three methods exist to determine the $3 \mathrm{D}$ of a scene. The first is based on the acquisition of a tomographic images sequence [6]. The second uses the analysis of intensity profile according to the visualized scene depth [7]. In both cases, the information is processed to have the $3 \mathrm{D}$ scene. The third method independently measures the time of flight of a round trip of the laser pulse between the object in the scene and the imager for each pixel [8]. Its reading mode, reducing the images frequency, especially when the pixels number is significant, limits certain applications when the speed is a very important factor. Furthermore, if particles (rain, fog) are in front of the imager, the measured distance does not correspond to the object distance in the scene but to that of particles. Conversely, MAITODIC assumes that the time of flight is known and only the information arriving at this precise moment is taken into account. Each time measurement value corresponds to a distance but also to a depth code (color) that will appear in the $3 \mathrm{D}$ image as the location of objects situated at this range. The scene will so be scanned in depth by slice, by modulating the known time of flight.

The architecture of the sensor is depicted in Fig 1. Every active pixel of the MAITODIC matrix operates independently and is driven by a common sequencer. They are equipped with a single optoelectronic sensor, an integrator, a comparator and a memory. So at the desired time of flight, if the retro- 


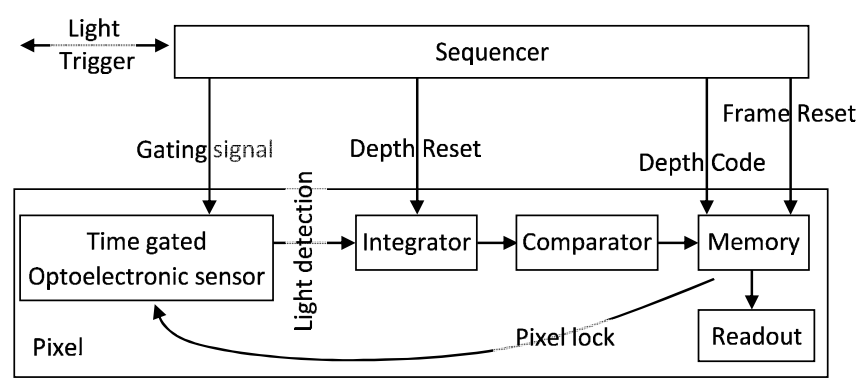

Fig. 1. Synoptic of the MAITODIC sensor with its pixel architecture

reflected light is intense enough compared with a threshold value, meaning that there is actually a reflection on an object, the pre-selected distance is memorized and the pixel is locked. Otherwise, the next time of flight is expected. A blocked buffer stays in this state until the next $3 \mathrm{D}$ recording of the scene. When the entire scene is scanned, all the buffers can be transferred at once towards the image memory, which can be read during the next $3 \mathrm{D}$ recording of the scene. The image frequency is not penalized by the number of pixels during the acquisition process. In case the intensity of a single pulse by slice cannot be enough to obtain an integrated signal level above the threshold value, it is possible to accumulate several laser pulses and, contrary to the usual time of flight measurements, to increase the number of pulses for the same slice within the limits of the image frequency. On the other hand, it is not necessary to have the same number of pulses by visualized slice. So furthest slices can receive more pulses than the closer one.

\section{CMOS SENSOR DESIGN}

This new architecture of real time 3D video sensor has been integrated within a CMOS sensor realized in a standard $180 \mathrm{~nm}$ technology. The different blocks are described below.

\section{A. Sequencer}

The sequencer generates the signals to drive the pixels of the sensors. It adjusts the delay between the light trigger and the gating signal of the pixel in order to choose the spatial slice depth according to the time of flight of the photon for the round trip in the considered medium (generally the air). In the same way, the width of the gating signal is related to the thickness of this spatial slice. At the beginning of a 3D frame, the sequencer resets all the pixels by activating the Frame Reset signal. To measure a signal at a given spatial slice, a depth code, which is in reality a specific voltage, corresponding to this spatial slice is set on $\mathrm{V}_{\text {DEPTH}}$. Then the sequencer order a predefined number of light pulse shots and temporal gates with a fixed delay. This one can be adjusted in regards of the expected returned signal strength and can be, for instance, increased for the very long distances where a photon is not necessarily detected at each laser pulse. If a pixel detects a sufficient number a photon, meaning that there is an object at this distance that reflect the light, the pixel locks automatically and samples the preset $\mathrm{V}_{\text {DEPTH. Subsequently, the sequencer }}$ chooses another spatial slice and operates a new burst of laser pulse, etc.
All the measurement parameters such as the number of temporal slices, their thickness, their absolute position are completely defined by the sequencer and the number of $\mathrm{V}_{\text {DEPTH }}$ code that can be resolved by the pixel. This approach makes the system very versatile and usable for short and long ranges as well as fine and coarse $3 \mathrm{D}$ measurements.

At a light pulse repetition rate of $20 \mathrm{MHz}$ and a target of 256 numbers of spatial slices, the average numbers of photon events that can be counted for each slice is 78125 per second. Thus the Dark Count Rate (DCR) of the SPAD which is in the order of $1 \mathrm{kHz}$, is relatively insignificant. Moreover, the effective DCR is strongly reduced by the time gate operation of the system. If a video rate of 25 images per second is aimed, the average number of photon events per 3D image and per slice is thus 3125 , ensuring a very good signal to noise ratio mainly limited by the photon shot noise.

The light pulse repetition rate for long range measurements is generally limited to about $100 \mathrm{kHz}$ for a distance of $1.5 \mathrm{~km}$ for instance. In this case, the number of possible photon events per second is reduced to 390 events per slice, i.e. only 15 photon events per slice and per image at a video rate of 25 image per second. Thus, the pixel sensitivity and especially the integrator sensitivity must be sufficient to detect 15 events.

\section{B. Time gated optoeletronic sensor}

The number of photon that hit the sensor for long range measurements can be as weak as one photon per pixel and per laser pulse. Thus, a very sensitive sensor has to be used to be able to detect the optical signal. A Single Photon Avalanche Diode (SPAD) based optoelectronic front end has been chosen to provide the high sensitivity. Moreover, a SPAD can operate in gating mode by biasing the junction above or under the breakdown voltage. The detail of the optical front end is depicted in Fig. 2.

The SPAD is based on a $\mathrm{P}+/ \mathrm{N}_{\text {well }}$ junction with a double Pwell/STI guardring [9]. The transistors $\mathrm{Mp}_{\text {Quench }}$ and $\mathrm{Mn}_{\text {Quench }}$ act as a passive quenching resistor and active quenching switch respectively. Transistors $\mathrm{Mp}_{\text {Lock }}$ and $\mathrm{Mn}_{\text {Lock }}$ allows to lock the pixel when Pixel_lock signal is high by forcing the quenching of the SPAD and blocking the SPAD Reset. Indeed, when a pixel has detected a reflection and memorized the depth code, its front-end is disabled to lock this pixel and reduce power consumption by the way.

The MpPPAD_reset allows to reset the SPAD to a biasing above the breakdown voltage. Two delayed control signal are used in

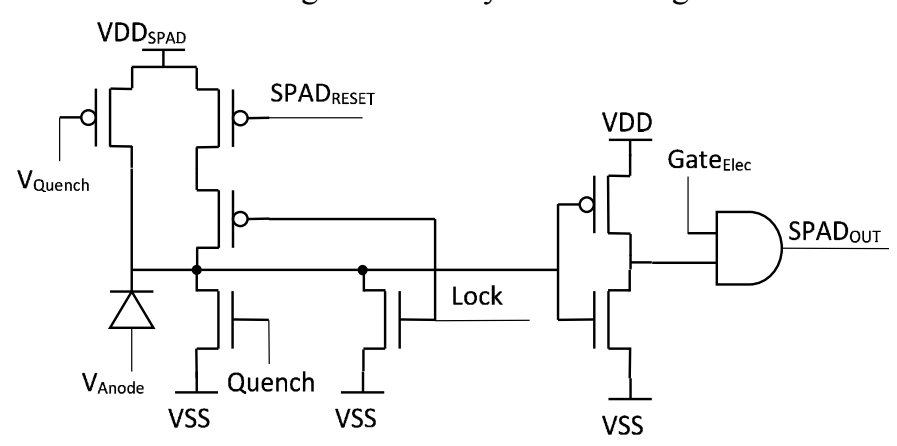

Fig. 2. Schematic of the time gated optoelectronic sensor 
order to generated subnanosecond reset time as described in [10]. $\mathrm{V}_{\text {anode }}$ voltage is tuned to set the SPAD excess voltage above the breakdown limit and the separate power supply VDD $_{\text {SPAD }}$ and VDD allows us to adjust the effective threshold of the $\mathrm{Mp}_{\text {comp }}$ and $\mathrm{Mn}_{\text {comp }}$ inverter based comparator.

If the SPAD is triggered, the cathode voltage decreases and is detected by the comparator setting its output to high. The Gate $_{\text {Elec }}$ gating signal generated according the technique described in [10] is then used to gate the comparator signal. At first order, the gate width is given by the time between the effective reset of the SPAD to the end of the Gate Elec $_{\text {signal. }}$ Temporal width as short as 200 ps has been reporter in [10]

\section{Integrator and comparator}

The integrator follows the time gated front-end and is based on a charge transfer process [11] and depicted in Fig. 3. The nodes $\mathrm{N}_{2}$ and $\mathrm{N}_{1}$ are reset to VDD at each new depth measurement and each laser pulse respectively. After each generated time gate, the $\mathrm{G}_{\mathrm{TX}}$ signal, typically in the range of 1 volt to 1.4 volts potentially allows us to transfer a part of the $\mathrm{N}_{1}$ charge into node $\mathrm{N}_{2}$ regarding the voltage of node $\mathrm{N}_{1}$. The first model of the circuit can be explained by assuming the parasitic capacitances $\mathrm{C}_{1}$ of node $\mathrm{N}_{1}$ and $\mathrm{C}_{2}$ of $\mathrm{N}_{2}$. The $\mathrm{Mn}_{\mathrm{GTX}}$ allows to transfer charges from node $\mathrm{N}_{1}$ and node $\mathrm{N}_{2}$ until its $\mathrm{V}_{\mathrm{GS}}$ voltage is above the threshold voltage $\mathrm{V}_{\mathrm{TH}}$. As the charges transfer is operating, the $\mathrm{N}_{1}$ voltage increase and turn off $\mathrm{Mn}_{\mathrm{GTX}}$ when the $\mathrm{VG}_{\mathrm{TX}}$ to $\mathrm{N}_{1}$ voltage reach $\mathrm{V}_{\mathrm{TH}}$. The charges Q1 transferred to node $\mathrm{N}_{2}$ during this process is then given by:

$$
Q_{1}=C_{1} \cdot\left[\left(V_{G_{T X}}-V_{N_{1}}\right)-V_{T H}\right]
$$

Following the injection of the $Q_{l}$ charges, the voltage of node $\mathrm{N}_{2}$ decreases. If the voltage of node $\mathrm{N}_{1}$ is initially biased above $\mathrm{VG}_{\mathrm{TX}}-\mathrm{V}_{\mathrm{TH}}$, no charge transfer occurs. Thus resetting node $\mathrm{N}_{1}$ to VDD ensure that the $\mathrm{N}_{2}$ is not affected until the transistor $\mathrm{Mn}_{\text {SPADOUT }}$ changes $\mathrm{N}_{1}$ voltage to $V_{\text {Gain }}$ voltage when a SPADout signal arise from the optoelectronic front-end meaning that a photon has been detected in the temporal gate. In this case, the $\mathrm{N}_{2}$ voltage decreased by a quantum $\Delta V_{Q}$ given by:

$$
\Delta V_{Q}=\frac{C_{1}}{C_{2}} \cdot\left[\left(V_{G_{T X}}-V_{\text {Gain }}\right)-V_{T H}\right]
$$

A second order approach shows that the longer the width of the $\mathrm{G}_{\mathrm{TX}}$ signal, the higher the absolute value of $\mathrm{V}_{\mathrm{Q}}$. Indeed, the transistor still transfer charges when operating in the subthreshold mode. Therefore, $V_{Q}$ can be adjusted thanks to $\mathrm{V}_{\mathrm{GAIN}}$ and the $\mathrm{G}_{\mathrm{TX}}$ duration to a few tens of $\mathrm{mV}$ down to a few $\mu \mathrm{V}$ allowing to reach the threshold of the output inverter with

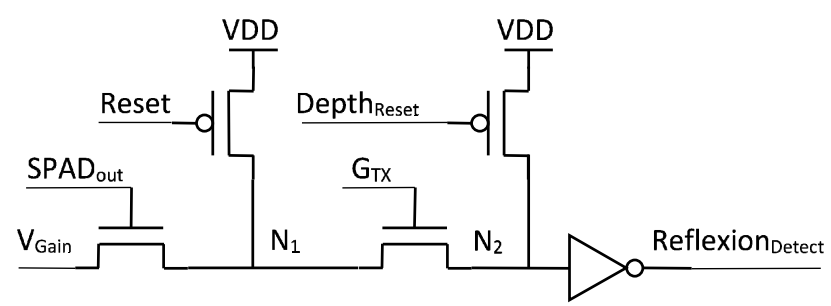

Fig. 3. Schematic of the integrator and the comparator a number of detected photon in the range from 10 up to 10000 detection events.

\section{Memory and readout}

The memory unit is depicted in Fig. 4. It includes a RS memory, a digital demultiplexer with an unselected output set to ' 1 ' and a selected output following the input, two PMOS based sampling cell that sample the $\mathrm{V}_{\text {DEPTH }}$ signal into the

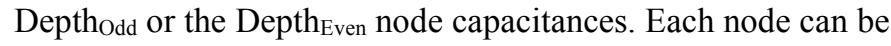
readout as a classic $3 \mathrm{~T}$ video imager on the column bus by NMOS based source followers that can be selected by the Selodd or Sel Even signals and a current source at the column bottom.

A new 3D frame starts with the reset of the RS memory by setting the Frame Reset signal. As a consequence, the output $\bar{Q}$ is set to ' 0 '. Depending on the selected frame with the Frame $_{\text {Sel, }}$ the corresponding sampling cell is then on, i.e. the sampling capacitance voltage is following $\mathrm{V}_{\text {DEPTH. Meanwhile, }}$ the unselected sampling cell is off, i.e. it memorizes the previous sampled value. This feature allows the readout of the previous frame (Odd numbers for instance) while the acquisition of the current is being operated (Even numbers for instance).

During the measurement of a given spatial slice, its corresponding depth code is set with the $\mathrm{V}_{\text {DEPTH }}$ voltage. If a reflection is measured at this slice, the Refexion Detect $_{\text {signal is }}$ kept inactive and thus has no effect on the sampling cell behavior. In other cases, as soon as the Reflexion Detect $_{\text {signal }}$ arises, the RS memory is set on and the selected frame sampling cell samples and hold the current $\mathrm{V}_{\text {DEPTH. At }}$. At the same moment, the lock signal is set to ' 1 ', then the optoelectronic front-end is locked, i.e. the SPAD is always quenched and as a result can't detect any new photon.

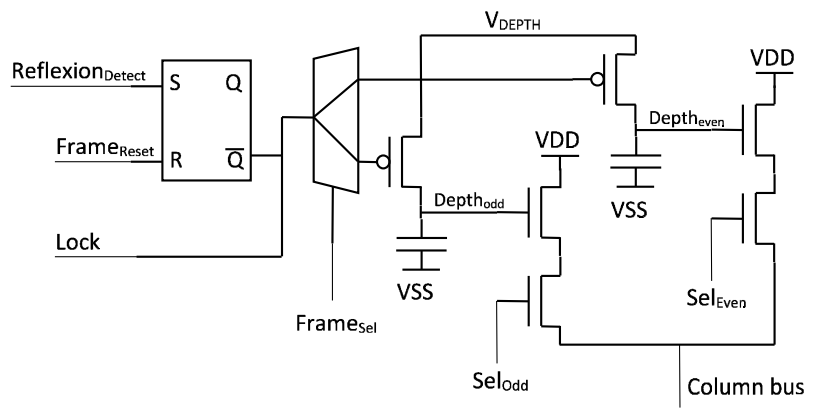

Fig. 4. Schematic of the memory unit and the readout

\section{LAYOUT}

The layout of a single pixel is depicted in Fig. 5. The pixel pitch is $36 \times 43 \mu \mathrm{m}^{2}$. Some additional decoupling capacitances have been added within the pixel to ensure good operation while switching the SPAD on/off. The diameter of the SPAD active area is $15 \mu \mathrm{m}$ which leads to a fill factor of more than $11 \%$. The routing of the high speed signal has been treated with particular attention to ensure a good propagation. The technique described in [10] allows us to compensate the skew to guarantee a sub nanosecond synchronicity. 


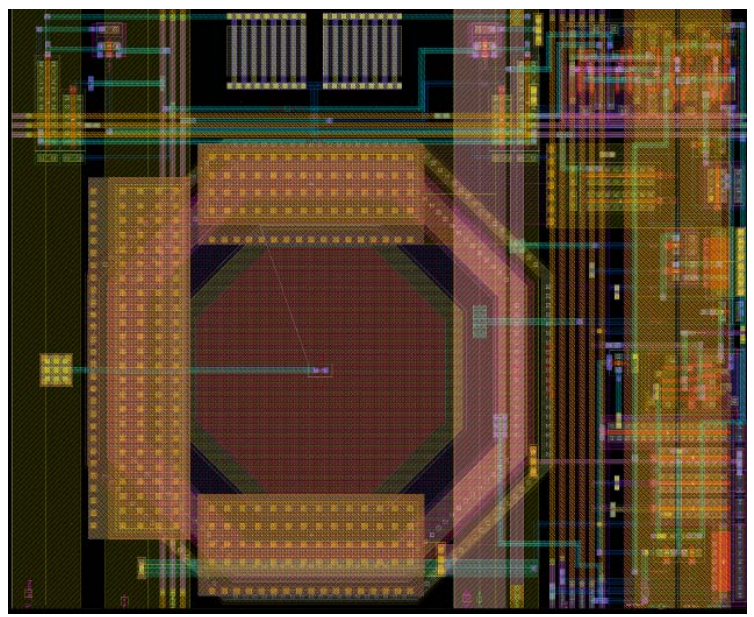

Fig. 5. Layout of a single pixel

V. SimULATION RESUlTS

To illustrate the functionality of the sensor, the simulation of a depth measurement with a systematical photon detection is shown in Fig. 6. The simulation begin with an active Frame $_{\text {Reset }}$ and a Depth Reset.

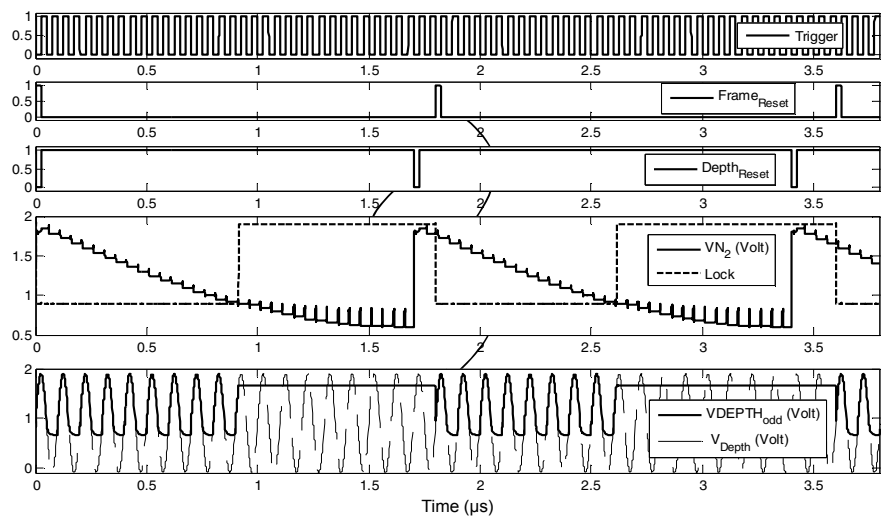

Fig. 6. Simulation results of a pixel detection, integration and locking

An arbitrary sinusoidal signal is applied to $V_{\text {Depth }}$ to demonstrate that the pixel locked when a sufficient number of reflection has been detected. Indeed at the $15^{\text {th }}$ trigger, at $0.9 \mu \mathrm{s}$, the integration node $\mathrm{VN}_{2}$ reach the 0.9 Volts comparator threshold thus inducing the pixel Lock. Before the lock, the sampling voltage node Depth ${ }_{\text {odd }}$ follows the $\mathrm{V}_{\text {DEPTH }}$ voltage and after the lock, its last value is sampled and held. At $1.7 \mu \mathrm{s}$, a Dept $t_{\text {Reset }}$ reset the integration node to VDD. At $1.8 \mu \mathrm{s}$, the Frame Reset signal unlocks the pixel. In normal operation mode, the FrameReset is applied once per frame and

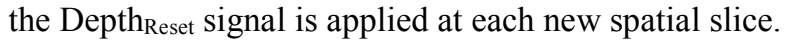

\section{CONCLUSION}

A Real time 3D video sensor with a time gated optical frontend and an active pulse light source is presented. The main characteristics of the sensor are summarize in table I. It offers several advantages with regard to the vacuum tube based image intensifier like the compactness. The photon counting capability of the sensor makes it possible to operate at a very long range of up to few kilometers through scattering environments. All pixels operate independently and in a fully parallelized process that makes it possible to handle more than 1000 Giga photon event assuming a $256 \times 256$ pixels array at a light pulse repetition rate of $20 \mathrm{Mhz}$. The sensor directly generates a 3D video image at a frame rate of 25 images per second or more without the need of a heavy post processing [12]. Concerning the long range 3D single photon imaging performed by a time gating approach with a SPAD [13], to the author's knowledge, it is the first time a SPAD based smart pixel that directly generates the $3 \mathrm{D}$ image is presented.

TABLE I. SENSOR CHARACTERISTICS

\begin{tabular}{|l|l|}
\hline Parameters & Value \\
\hline Temporal gate, Depth resolution & Down to $200 \mathrm{ps}, 3 \mathrm{~cm}$ in the air \\
\hline Pixel size & $36 \times 43 \mu \mathrm{m}^{2}$ \\
\hline Acquisition range & From a few cm up to several $\mathrm{km}$ \\
\hline Light pulse repetition rate & $\mathrm{Up}$ to $20 \mathrm{MHz}$ \\
\hline Pixel transistor count & 46 \\
\hline SPAD Diameter - Fill factor & $15 \mu \mathrm{m}-11 \%$ \\
\hline
\end{tabular}

\section{REFERENCES}

[1] E. Repasi, P. Lutzmann, O. Steinvall, M. Elmqvist, B. Göhler and G. Anstett, "Advanced short-wavelength infrared range-gated imaging for ground applications in monostatic and bistatic configurations," Appl. Opt., vol. 48 (31), pp. 5956-5969, 2009.

[2] G. R. Fournier, D. Bonnier, J. L. Forand and P. W. Pace, "Range-gated underwater laser imaging-system," Opt. Eng., 32, pp. 2185-2190, 1993.

[3] M. Laurenzis, F. Christnacher and D. Monnin, "Longe-range threedimensional active imaging with super-resolution depth mapping," Opt. Lett., vol. 32 (21), pp. 3146-3148, 2007.

[4] E. Belin, F. Christnacher, F. Taillade and M. Laurenzis, "Display of an analytical model for backscattered luminance and a full-field range gated imaging system for vision in fog," San Diego/CA, US, vol. 7088, 2008.

[5] A. Matwyschuk, 3D imaging method 20140528 (FR).

[6] J. Busck and H. Heiselberg, "Gated Viewing and High-Accuracy Threedimensional Laser Radar," App. Optics, vol. 43 (24), pp. 4705-4710, 2004.

[7] M. Laurenzis, F. Christnacher, N. Metzger, E. Bacher and I. Zielenski, "3d range-gated imaging at infrared wavelengths with super-resolution depth mapping," Proc of SPIE. vol. 7298, 2009.

[8] http://www.mesa-imaging.ch/home/.

[9] I. Malass, W. Uhring, J-P. Le Normand, N. Dumas, F. Dadouche, "Evaluation of size influence on performance figures of a Single Photon Avalanche Diode fabricated in a $180 \mathrm{~nm}$ standard CMOS technology," AICSP, pp. 1-8, 2016, doi:10.1007/s10470-016-0763-8

[10] O. Maciu, W. Uhring, J-B. Kammerer, J-P. Le Normand, N. Dumas, F. Dadouche, "Sub-Nanosecond Gated Photon Counting for High Spatial Resolution CMOS Imagers," IEEE NEWCAS pp. 1-4, november 2016, doi:10.1109/NEWCAS.2016.7604816

[11] F. Morel , J-P. Le Normand, V. Zint, W. Uhring, Y. Hu , D. Mathiot, “A new spatiotemporal CMOS imager with analog accumulation capability for nanosecond low-power pulse detections," IEEE Sensors Journal, pp. 1200-1208, Vol. 6, 2006, doi:10.1109/JSEN.2006.881356

[12] Z. Li, X. S. Ye, H. Zhang, L. Lu, C. Lu and L. C. Cheng, "Real-time 3D video system based on FPGA," 2013 3rd Int. Conf. on CECN, Xianning, 2013, pp. 469-472. doi: 10.1109/CECNet.2013.6703371

[13] A. M. Pawlikowska, R. M. Pilkington, K. J. Gordon, P. A. Hiskett, G, S. Buller, R. A. Lamb, "Long-range 3D single-photon imaging lidar system," Proc. of SPIE 9250, 925007, November 2014, doi: $10.1117 / 12.2066837$ 